\title{
Electrifying long-range signalling
}

\begin{tabular}{l} 
Pf \\
potassium \\
ion channels \\
are used to \\
propagate \\
electrical \\
signals \\
throughout \\
Bacillus \\
subtilis \\
biofilms \\
\multicolumn{1}{c}{}
\end{tabular}

Bacteria contain many classes of ion channels, but their functions in bacterial physiology have largely remained a mystery. Now, Prindle et al. show that potassium ion channels are used to propagate electrical signals throughout Bacillus subtilis biofilms in a long-range process that coordinates the metabolic responses of the community.

Previous studies demonstrated that B. subtilis biofilms undergo periodic metabolic oscillations that are triggered by nutrient availability. When cells in the interior of the biofilm are unable to access glutamate, an amino acid that is essential for bacterial growth, these cells shut down their production of ammonium, a metabolite that is required for growth by the cells in the periphery. This limitation in the availability of ammonium arrests cell growth in the periphery, which enables glutamate to reach the interior cells, which in turn restores growth of the cells in the interior of the biofilm. These cells resume their production of ammonium, enabling growth of the peripheral cells. However, how

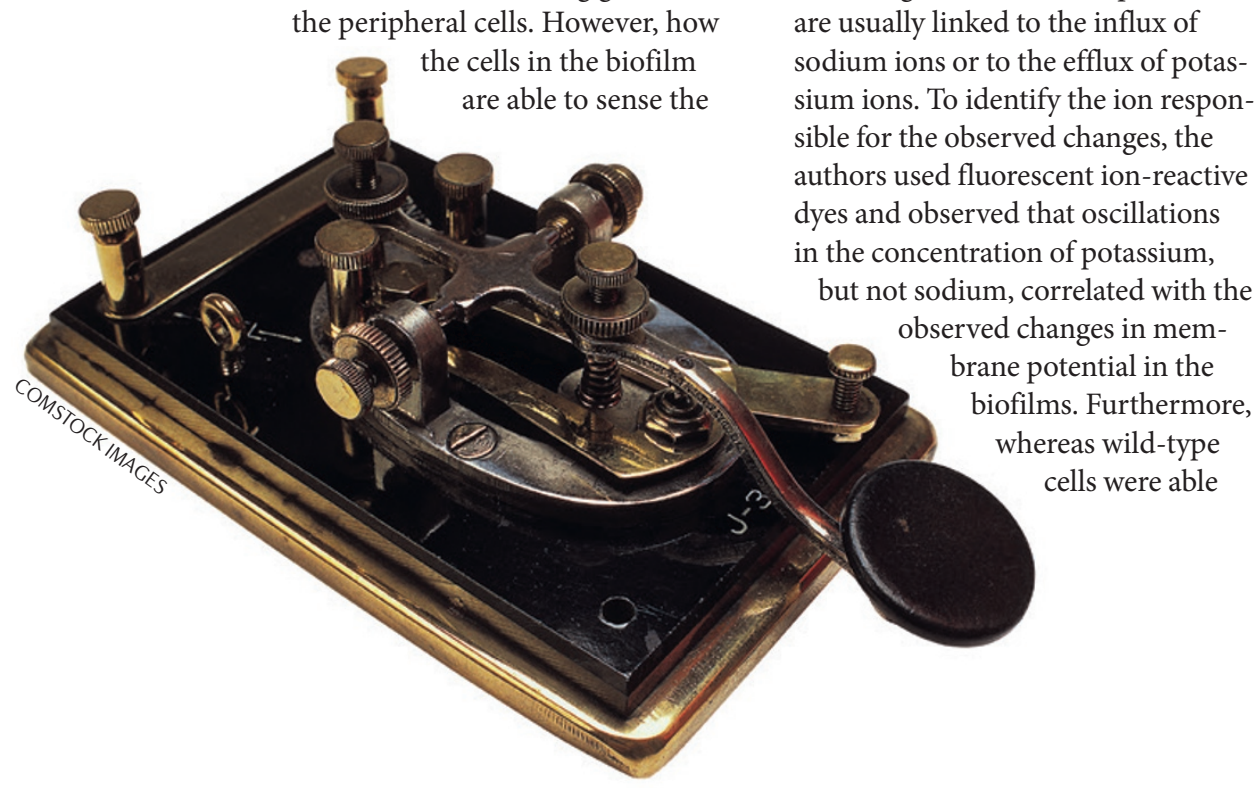

metabolic status of the entire community is unknown.

Because glutamate and ammonium are both charged metabolites, the intracellular levels of which are regulated by the transmembrane potential of the cell, the authors hypothesized that changes in electrochemical signalling could be responsible for regulating the metabolic status of the biofilm. To investigate this, they used a fluorescent cationic dye to measure the membrane potential within biofilms that were grown in microfluidic devices. Notably, they observed periodic oscillations in membrane potential throughout the biofilm that coincided with the metabolic oscillations that had been previously described. Furthermore, supplementation of the growth medium with glutamine, which bypasses the need for glutamate and ammonium for biofilm growth, eliminated the observed oscillations in membrane potential, suggesting that membrane potential regulates the metabolic oscillations.

Changes in membrane potential are usually linked to the influx of sodium ions or to the efflux of potassium ions. To identify the ion responsible for the observed changes, the -reactive dyes and observed that oscillations in the concentration of potassium, sodium, correlated with the ved changes in memiofilms. Furthermore, reas wild-typ to export potassium when deprived of glutamate (a condition that triggers the metabolic response), cells lacking yugO - the gene encoding the only potassium channel that has been experimentally described in B. subtilis - were unable to do so. Notably, wild-type cells were also able to propagate potassium signalling when treated with transient bursts of potassium, whereas cells lacking YugO could not. Collectively, these data suggest that YugO is responsible for the initiation and the propagation of the potassium signal throughout the biofilm.

Combining these observations with mathematical modelling, the authors propose a model by which metabolically stressed cells in the interior of the biofilm (which lack access to glutamate) release intracellular potassium, which alters the membrane potential of neighbouring cells. This change in membrane potential reduces the ability of the neighbouring cells to take up glutamate, which results in metabolic stress causing further potassium release, which propagates a 'wave of depolarization' that sustains potassium signalling throughout the biofilm. This signalling wave from the interior cells to the periphery ultimately limits the ability of the peripheral cells to access glutamate, enabling the interior cells to gain access to this amino acid.

These data demonstrate a physiological role for bacterial ion channels in the coordination of metabolic responses by enabling long-range communication within biofilms. Cláudio Nunes-Alves 